\title{
Design and Performance Analysis of a Multi-User OFDM Based Differential Chaos Shift Keying Communication System
}

\author{
Georges Kaddoum*
}

\begin{abstract}
In this paper, a Multi-user OFDM based Chaos Shift Keying (MU OFDM-DCSK) modulation is presented. In this system, the spreading operation is performed in time domain over the multi-carrier frequencies. To allow the multiple access scenario without using excessive bandwidth, each user has $N_{P}$ predefined private frequencies from the $N$ available frequencies to transmit its reference signal and share with the other users the remaining frequencies to transmit its $M$ spread bits. In this new design, $N_{P}$ duplicated chaotic reference signals are used to transmit $M$ bits instead of using $M$ different chaotic reference signals as done in DCSK systems. Moreover, given that $N_{P}<<M$, the MU OFDM-DCSK scheme increases spectral efficiency, uses less energy and allows multiple-access scenario. Therefore, the use of OFDM technique reduces the integration complexity of the system where the parallel low pass filters are no longer needed to recover the transmitted data as in multicarrier DCSK scheme. Finally, the bit error rate performance is investigated under multi-path Rayleigh fading channels, in the presence of multi-user and additive white Gaussian noise interferences. Simulation results confirm the accuracy of our analysis and show the advantages of this new hybrid design.
\end{abstract}

Index Terms-Non-coherent spread spectrum communication system, multiple access, OFDM-DCSK, energy efficiency, performance analysis.

\section{INTRODUCTION}

$\mathbf{T}$ HE multiple access direct sequence spread spectrum (DS$\mathrm{SS}$ ) system is known to have the ability to combat multipath interference and to survive in frequency selective channels [1]. Therefore, the capacity of this system is limited by the multiple access interference (MAI) and the inter-chip interference in the presence of multipath frequency selective fading. The combination of the DS-SS system with OFDM modulation reduces significantly the inter-chip interference in frequency selective channels and enhances the spectral efficiency of the system. Therefore, several combinations of multi-carrier transmission and Code Division Multiple Access (CDMA), like Multi-Carrier CDMA (MC-CDMA), MultiCarrier Direct-Sequence CDMA (MC-DS-CDMA) and Orthogonal Frequency Code Division Multiplexing (OFCDM) are proposed in the literature [2], [3], [4]. In MC-CDMA, one-bit chips are spread over $N$ subcarriers in the frequency domain [2], while for MC-DS-CDMA, time and frequency

G. Kaddoum is with University of Québec, ÉTS, LaCIME Laboratory, 1100 Notre-Dame west, H3C 1K3, Montreal, Canada (e-mail: georges.kaddoum@etsmtl.ca)

* This work has been supported by the NSERC discovery grant $435243-$ 2013. spreading are used [4]. Time-domain spreading is employed to increase the processing gain in each subcarrier signal, while frequency domain spreading is used to increase the total processing gain.

The chaotic signal has a sensitive dependence upon initial conditions property that allows the generation of a theoretical infinite number of uncorrelated signals with excellent correlation properties. These signals have been shown to be well suited for spread-spectrum modulation because of their inherent wideband characteristic [5] [6] [7] and their sharp auto correlation and low cross correlation values [8].

Various digital chaos-based communication schemes have been evaluated and analysed including coherent chaos-shiftkeying (CSK) [5], [9], [10], chaos-based DS-CDMA [8], [6], [7] and non-coherent Differential Chaos Shift Keying (DCSK) [11], [12], [13], [14]. In CSK and chaos-based DSCDMA, chaotic sequences are used instead of conventional spreading codes to spread data signals. The later is used in DSCDMA. The usage of chaotic sequences enhances the security and the performance of the transmission [6] but such a scheme would require the generation and the synchronization of the chaotic sequence at the receiver side which is non-trivial. For instance, the chaotic synchronization proposed by Pecora and Carroll in [15] is still practically impossible to achieve in a noisy environment and, as a result, the coherent system can not be used in realistic applications.

Additionally, the DCSK modulation is chosen in this paper for its various advantageous. Beside benefiting from the excellent correlation properties of chaotic signals, the demodulation process for such non-coherent systems can be carried out without the generation of neither chaotic signals nor the use of any channel estimators [11], [12], [13], [14], [16] which makes this system easy to implement [17]. Therefore, the common points between DCSK and differential phase shift keying (DPSK) modulation is that both are non-coherent schemes and do not require channel state information at the receiver to recover the transmitted data [1], [18], [16]. However, DCSK systems are more robust to multipath fading environments than DPSK schemes [18] and are suitable for Ultra-Wide band (UWB) applications [19], [20], [16], [18], [21].

In DCSK, each bit duration is divided into two equal slots. In the first slot, a reference chaotic signal is sent. Depending on the bit being sent, the reference signal is either repeated or multiplied by the factor of -1 and transmitted in the second slot. The performance of the DCSK communication systems under different scenarios and with other transmission 
technique has been evaluated in [11], [12], [13], [14], [21] and [22]. The significant drawback of DCSK are the fact that half the bit duration is spent sending non-informationbearing reference samples [5] and that it depends on wideband delay lines that are very difficult to implement in the current CMOS technology [23], [24]. These two points are serious data rate reducers that also introduce energy-inefficiency into the system.

To overcome the mentioned deficiencies of the DCSK scheme, a growing number of research has been conducted to propose new non-coherent systems. The high efficiency HEDCSK [25], reference modulated RM-DCSK [26], M-DCSK [27] and differentially DDCSK [28] are proposed to partially improve the performance of DCSK system, but at the cost of an increased system complexity. To reduce or avoid the use of delay lines in DCSK, a system called code-shifted CS-DCSK in which reference and data sequences are separated by Walsh code sequences instead of time delay multiplexing is proposed in [23]. An extended version of this scheme is presented in [24] in which the Walsh codes are replaced by different chaotic sequences to separate the data, and the reference signal is transmitted over an orthogonal frequency. These two methods increase the data rate and improve the bit error probability (BEP) but require the generation of chaotic or Walsh codes at the receiver which affects the non-coherent nature of the DCSK system.

Related works: In [19], the authors have proposed multicarrier DCSK (MC-DCSK) system, which was developed to support multi-user transmission in [29]. In this scheme a chaotic reference sequence is transmitted over a predefined subcarrier frequency while multiple modulated data streams are transmitted over the remaining subcarriers. The MC-DCSK scheme improves energy efficiency, offers increased data rates but requires the use of parallel matched filters and demands bandwidth.

Inspired by the MC-DCSK system presented in [19], the authors in [30] present an OFDM-DCSK system as an alternative solution to reduce the integration complexity of the multi-carrier DCSK system proposed in [29]. In that system, one chaotic reference signal is transmitted over the central subcarrier, while multiple modulated data streams are transmitted over the remaining subcarriers. The system proposed in [30] considers single-user transmission in AWGN channels only. Although the OFDM-DCSK system reduces the complexity of the MC-DCSK scheme, but with its present form, this system cannot support multiple access communication.

Contributions and paper outline: In this paper, we propose a solution to reduce the complexity of the MC-DCSK system [19] inspired by the system proposed in [30]. Our proposed system is a combination of an OFDM and DCSK modulations that reduces complexity, performs over fading channels and allows multiple access communication.To reach this end, from $N$ total subcarriers, each user of the $P$ users has $N_{P}$ private subcarriers and $N_{S}$ shared public subcarriers such that $N_{S}=N-P N_{P}$. The private subcarriers are used to transmit the reference signals of each individual user, while the public frequencies are shared with other users to carry the data slots. We propose that only $N_{P}$ chaotic reference signals be used to transmit $M$ bits instead of using $M$ reference signals as done in DCSK system, where $N_{P}<<M$. Following serial to parallel conversion and multicarrier demodulation to the baseband at the receiver, the reference signal is recovered and used to despread the transmitted bits.

Compared to DCSK system, just $N_{P}$ references are used to transmit $M$ bits, this operation saves the transmitted bit energy. Moreover, the distribution of the reference signals over the $N_{P}$ predefined private frequencies follows the combtype pattern design. In fact, the comb-type design allows the receiver to have a fast adaptation to the channel when this lattice changes in time from one OFDM symbol to another [31]. Furthermore, the multiple access remains possible in frequency domain for this non-coherent system by using $N_{p}$ private frequency for each user. Moreover, by comparing to MC-DCSK system, this scheme reduces complexity by using IFFT/FFT operations instead of parallel matched filters as done in [19], saves the bandwidth by using shared frequencies to transmit the data and solves the RF delay line problem mentioned in [23]. Further, the proposed MU OFDM-DCSK scheme benefits from the properties of DCSK system in terms of resistance to multipath interference and data recovery without the generation of chaotic signals or the use of complex channel estimators at the receiver.

Besides, we thoroughly analyse the BER performance under multipath Rayleigh fading and AWGN channels. In our approach of computation, the standard Gaussian approximation (SGA) is used to approximate the sum of multiple access interference (MAI) signals as an additive white Gaussian noise in addition to background noise [32], [33]. In this part of the paper, we derive the analytical bit error rate expressions and show the accuracy of our analysis by matching the numerical performance. The proposed system is commodious for Wireless Sensor Network (WSN) and ultra wide band applications [34], which have power limitations, evolve in harsh environments and exhibit high resistance to multipath interference.

The remainder of this paper is organized as follows. In section II, the DCSK system is presented briefly then the architecture of the MU OFDM-DCSK system architecture is explained and the energy efficiency of the system is examined. The performance of the system is derived in section III. Simulation results and discussions are presented in section IV and concluding remarks are presented in section V.

\section{Multi-USER OFDM-DCSK SyStem ARChiteCtURE}

\section{A. DCSK communication system}

We start this section by explaining the DCSK communication system in order to understand the novel extension parts of the proposed system and to use this as a comparative benchmark to illustrate the achieved performance enhancements.

As shown in Fig. 1, within the DCSK modulator, each bit $s_{i}=\{-1,+1\}$ is represented by two sets of chaotic signal samples, with the first set representing the reference, and the second carrying data. If +1 is transmitted, the data-bearing sequence is equal to the reference sequence, and if -1 is transmitted, an inverted version of the reference sequence is 


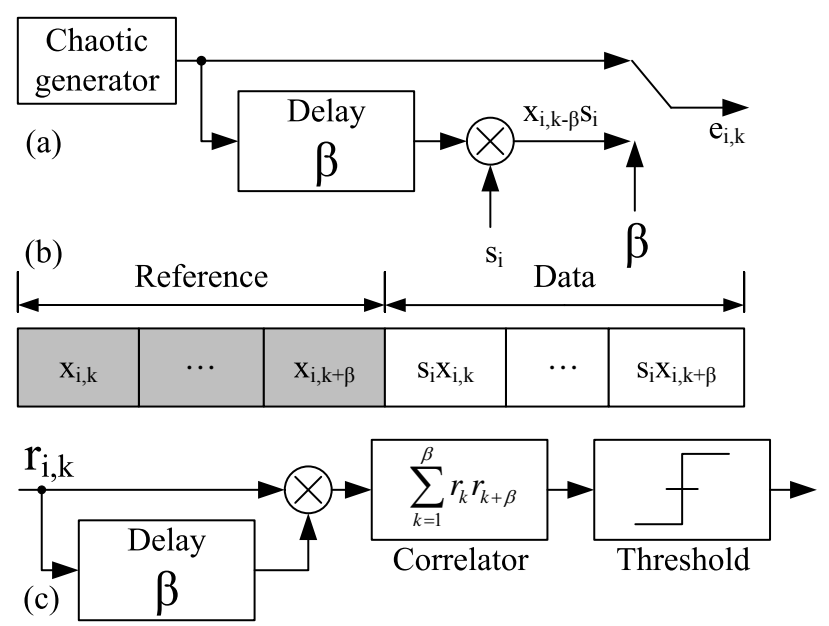

Fig. 1: Block diagram of the general structure of the DCSK communication system: (a) transmitter (b) frame (c) receiver.

used as the data-bearing sequence. Let $2 \beta$ be the spreading factor in DCSK system, defined as the number of chaotic samples sent for each bit, where $\beta$ is an integer. During the $i^{\text {th }}$ bit duration, the output of the transmitter $e_{i, k}$ becomes

$$
e_{i, k}= \begin{cases}x_{i, k} & \text { for } 1<k \leq \beta, \\ s_{i} x_{i, k-\beta} & \text { for } \beta<k \leq 2 \beta,\end{cases}
$$

where $x_{k}$ is the chaotic sequence used as reference and $x_{k-\beta}$ is the delayed version of the reference sequence $x_{k}$.

Fig. 1 illustrates that the received signal $r_{k}$ is correlated to a delayed version of the received signal $r_{k+\beta}$ and summed over a half bit duration $T_{b}$ (where $T_{b}=2 \beta T_{c}$ and $T_{c}$ is the chip time) to demodulate the transmitted bits. The received bits are estimated by computing the sign of the output of the correlator, as illustrated in Fig. 1 (c).

As shown in Fig. 1, half of the transmitted energy and half of the bit duration time are spent sending a non-informationbearing reference. Therefore, the data rate of this architecture is seriously reduced compared to other systems using the same bandwidth, leading to a loss of energy and spectral efficiency.

\section{B. Chaotic generator}

In this paper, a second-order Chebyshev polynomial function $(\mathrm{CPF})$ is employed

$$
x_{k+1}=1-2 x_{k}^{2} .
$$

This map is chosen for the easy way in which it generates chaotic sequences and the good performance [10]. In addition, chaotic sequences are normalized such that their mean values are all zero and their mean squared values are unity, i.e., $E\left(x_{k}\right)=0$ and $E\left(x_{k}^{2}\right)=1$.

\section{The MU OFDM-DCSK transmitter}

In this section we will present the MU OFDM-DCSK design. The aim of the proposed system is to reduce the hardware complexity of the MC-DCSK proposed in [19], to increase the data rate, to reduce the transmitted bit energy, to operate in multi-user scenario, to benefit from the properties of OFDM modulation and to perform without any need to RF delay circuits or complex channel estimators.

The structure of the modulator and the transmitted signal are shown in Fig. 2 and Fig. 3. In this system, we consider $N_{t}$ subcarriers among which $N$ subcarriers at the central spectrum are used for transmission and the remaining $N_{t}-N$ subcarriers which are located at the two edges of the spectrum form the guard band and the unused subcarriers $N_{u}$. In our scheme and for $P$ users, $P N_{P}$ frequencies out of $N$ subcarriers are used to transmit the $P$ different reference signals. The edges and the center of the spectrum are allocated to transmit the reference signals of different users and the remaining $N_{S}$ frequencies are shared to transmit the spread data. As shown in Fig. 3, the distribution of the reference signal over the predefined private frequencies follows the comb-type pattern design [31]. In fact, the comb-type design allows the receiver to have a fast adaptation to the channel when this lattice changes in time from one OFDM symbol to another. It is important to note that different uncorrelated reference signals of $P$ users are used in the same fashion as pilot signals spreading codes of the OFDM-DCSK system.

Therefore, with this design, only the reference signals (i.e pilots) of different users are separated in the frequency domain to allow multiple access communications. As shown in Fig. 3 , the spreading operation is done in the time domain. This will require $\beta$ number of IFFT operations to transmit the $M$ spread bits with a spreading factor of $\beta$. In addition, since each user shares a part of his bandwidth with the other users, this reduces the total required bandwidth but increases MAI. However, MAI can be reduced by increasing the spreading factor value. As shown in Fig. 3, the OFDM-DCSK symbol duration $T_{s}$ is given by

$$
T_{s}=N \beta T_{c}
$$

where $T_{O F D M}=N T_{c}$ is the time duration of OFDM symbol.

After each IFFT operation the parallel signal is converted into a serial sequence and a cyclic prefix is added to eliminate the intersymbol interference and to allow a simpler frequencydomain processing. Hence, the OFDM-DCSK system benefits from the non-coherent advantages of DCSK and the spectral high data rate of OFDM modulation. As shown in Fig. 2, the chaotic sequence $\mathbf{x}_{p}=\left[x_{1, p}, \ldots, x_{k, p}, \ldots, x_{\beta, p}\right]$ is transmitted over $N_{P}$ frequencies which is used as reference signal and spreading code for the $M$ bits of user $p$. Hence, the $M$ bits stream of user $p$ are spread due to multiplication in time with the same chaotic spreading code $x_{p}(t)$.

$$
x_{p}(t)=\sum_{k=1}^{\beta} x_{k, p} g\left(t-k T_{c}\right),
$$

where $\beta$ is the spreading factor, $g(t)$ is the shaping filter which is assumed to be rectangular in this paper and $T_{c}$ is the chip duration. 

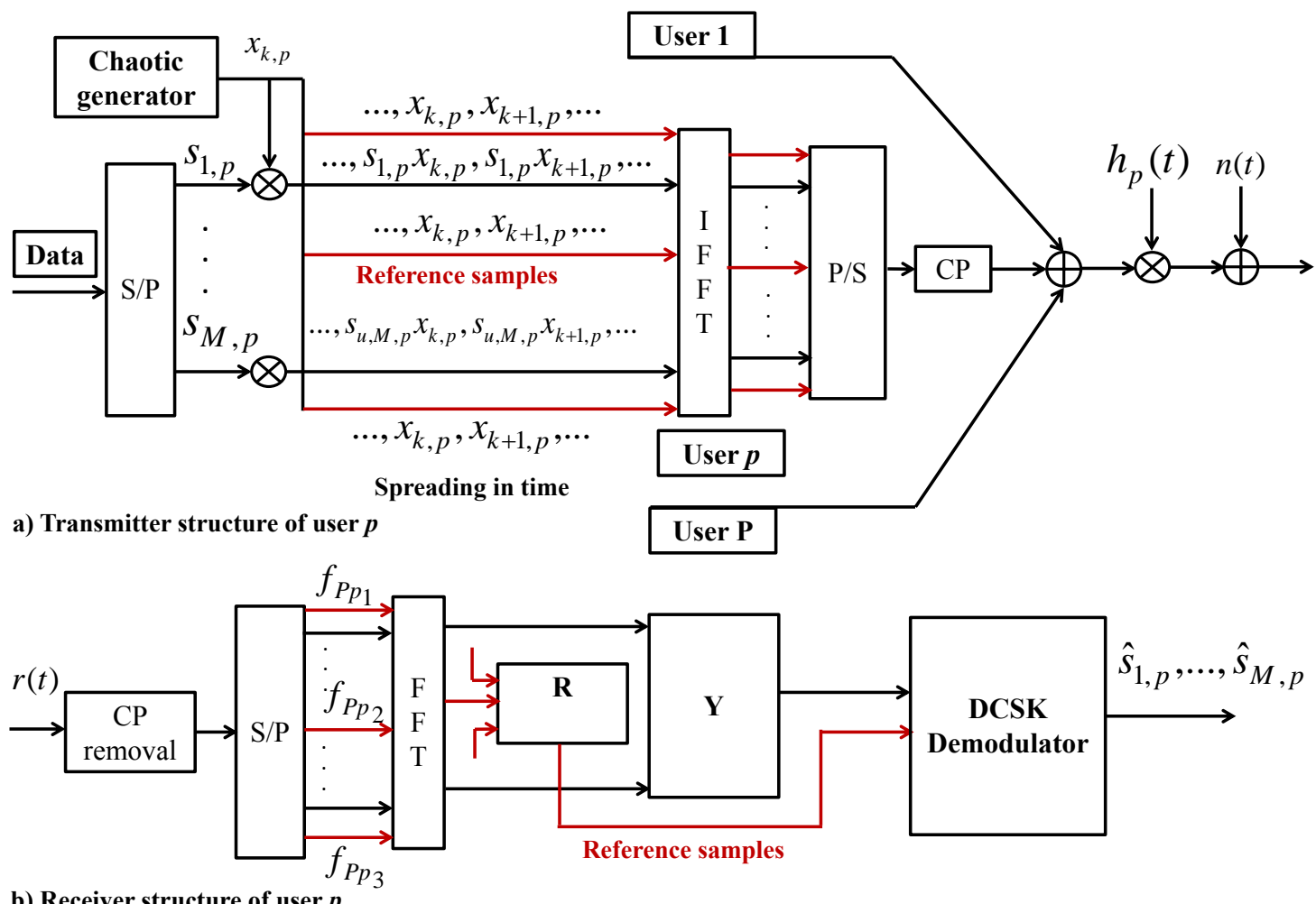

b) Receiver structure of user $p$

Fig. 2: Block diagram of the MU OFDM-DCSK system.

For simplicity, the insertion and removal of cyclic guard prefix or postfix is used in this system with period $\Delta$ but not expressed in our mathematical equations. Therefore, the transmitted signal of the $p^{\text {th }}$ user of OFDM-DCSK system is given by

$$
\begin{aligned}
& e_{p}(t)=\sum_{\nu=1}^{N_{P}} \sum_{k=1}^{\beta} x_{k, p} e^{2 \pi j f_{P_{p_{\nu}}}\left(t-k T_{c}\right)} g\left(t-k T_{c}\right)+ \\
& \sum_{\substack{i=1 \\
i \neq p}}^{M} \sum_{k=1}^{\beta} x_{k, p} s_{i, p} e^{2 \pi j f_{S_{p_{i}}}\left(t-k T_{c}\right)} g\left(t-k T_{c}\right),
\end{aligned}
$$

where $e_{p}(t)$ represents the transmitted OFDM symbol of user $p, f_{P_{p_{\nu}}}$ is its $\nu$ th private frequency used to transmit the reference chaotic signal $x_{k, p}, N_{P}$ is the number of private frequencies per user, $f_{S_{p_{i}}}$ is the $i^{\text {th }}$ shared public frequency of the $N_{S}=\left(N-P N_{P}\right)$ remaining public frequencies to transmit the $i^{\text {th }}$ bit of the $M$ block of bits. Hence, the maximal number of transmitted bits per user must be equal to the number of shared frequencies $N_{S}$, (i.e. $M \leq N_{S}$ ). As described mathematically in the above formula, the spreading operation is done in time domain where $\beta$ number of IFFT operations are required to transmit an OFDM-DCSK symbol of $N_{P}$ reference signals with $M$ spread bits. Finally, for a given number of users $P$, the maximum number of allowed subcarriers to transmit the data would be

$$
N_{S}=N_{t}-N_{c p}-N_{u}-P N_{P},
$$

where $N_{c p}$ and $N_{P}$ represent the number subcarriers dedicated to transmit the cyclic prefix and the pilot signal respectively and $N_{u}$ represents the number of unused subcarriers which is defined according to the used standards (i.e. $N=N_{t}-N_{c p}-$ $\left.N_{u}\right)$.

It is assumed that the OFDM-DCSK signal is transmitted over a multipath fading channel, the equivalent impulse response of the channel for the $p^{\text {th }}$ user is

$$
h_{p}(t)=\sum_{l=1}^{L_{p}} \sum_{k=1}^{\beta} \alpha_{p, l,\left\lceil\frac{k N T_{c}}{T_{h}}\right\rceil}(t) \delta\left(\tau-\tau_{p, l}\right),
$$

where $T_{h, p}=\chi_{p} N T_{c}$ is the time where the channel coefficient $\alpha_{p}$ is maintained constant during the transition of $\chi_{p}$ OFDM symbols of user $p$ and $\lceil$.$\rceil is the ceiling operator.$

In our paper the complex channel coefficients are zero mean and follow Rayleigh distribution given by

$$
f(\alpha \mid \sigma)=\frac{\alpha}{\sigma^{2}} e^{-\frac{\alpha^{2}}{2 \sigma^{2}}}, \quad \alpha \geq 0,
$$

where $\sigma>0$ is the scaling factor of the distribution representing the root mean square value of the received voltage signal before envelope detection.

The received MU OFDM-DCSK signal over the wireless channel is given by

$$
r(t)=\sum_{p=1}^{P} h_{p}(t) \otimes e_{p}(t)+n(t),
$$

where $P$ is the total number of users, $\otimes$ is the convolution operator and $n(t)$ is a circularly symmetric complex Gaussian noise with zero mean and power spectral density of $N_{0}$. 


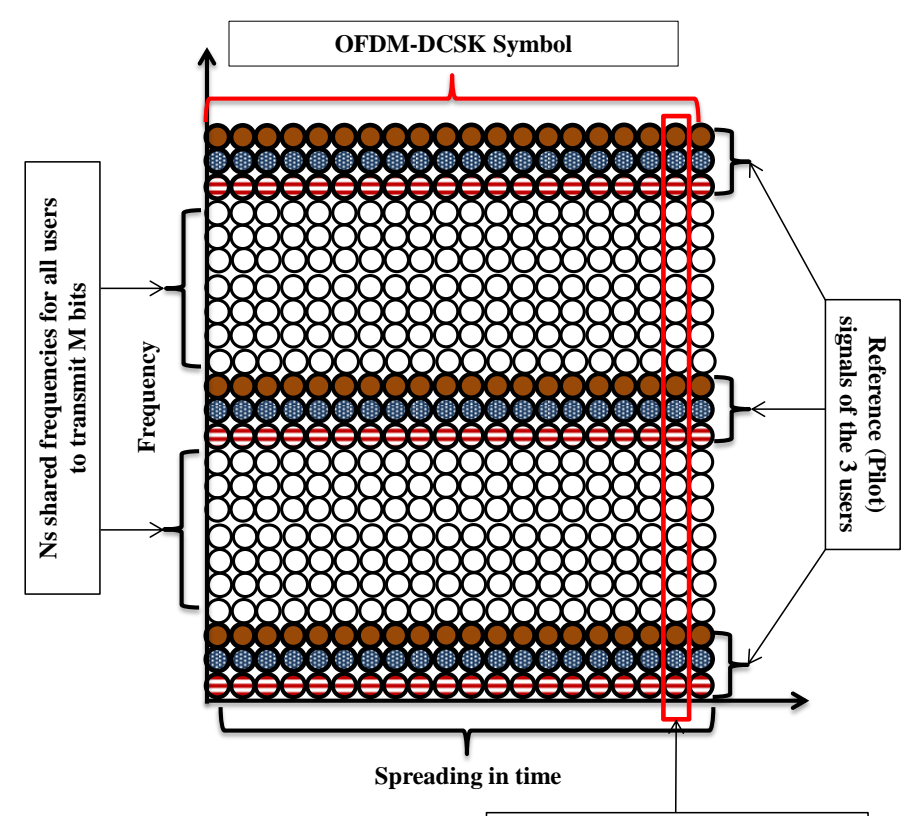

OFDM symbol of $\mathrm{N}$ subcarriers

Fig. 3: Signal structure with comb-type reference sequences for the $p^{\text {th }}$ user.

\section{Energy efficiency}

In this section we analyse the energy efficiency of the OFDM-DCSK system. In fact, for the conventional DCSK system, a new reference signal is transmitted with every bit. Hence, the total required bit energy $E_{b}$ to transmit one bit for a conventional DCSK system is

$$
E_{b}=E_{\text {data }}+E_{\text {ref }}
$$

where $E_{\text {data }}$ and $E_{\text {ref }}$ are the energies required to transmit data and reference signals respectively. Without loss of generality, for DCSK system, data and reference energies could be equal such that

$$
E_{\text {data }}=E_{\text {ref }}=T_{c} \sum_{k=1}^{\beta} x_{k}^{2} .
$$

Then for DCSK systems, the transmitted energy $E_{b}$ for a given bit becomes

$$
E_{b}=2 T_{c} \sum_{k=1}^{\beta} x_{k}^{2}=2 E_{\text {data }} .
$$

As shown in Fig. 3, each $M$ bits of the proposed OFDMDCSK system require $N_{P}$ replicas of the chaotic reference signal where $N_{P}<M$. Hence, $N_{P}$ multiples of the reference energy $E_{\text {ref }}$ are required to transmit $M$ bits. Thus, the energy required to transmit one bit of OFDM-DCSK system becomes

$$
E_{b}=E_{\text {data }}+\frac{N_{P} E_{\text {ref }}}{M} .
$$

It is clear from (13) that $E_{b}<2 E_{\text {data }}$ in the OFDM-DCSK system. Furthermore, equation (13) may be expressed as

$$
E_{b}=\left(\frac{M+N_{P}}{M}\right) T_{c} \sum_{k=1}^{\beta} x_{k}^{2}
$$

and in terms of $E_{\text {data }}$ as

$$
E_{b}=\left(\frac{M+N_{P}}{M}\right) E_{\text {data }} .
$$

Therefore, OFDM-DCSK modulation adds a cyclic prefix which requires extra energy. Hence, the total energy consists of the bit energy $E_{b}$ and the energy of the cyclic prefix $E_{c p}$. Since cyclic prefix is a partial copy of the IFFT output, the total energy $E_{t o t}$ of the OFDM-DCSK symbol can be calculated as

$$
E_{\mathrm{tot}}=E_{b}+E_{c p}
$$

where $E_{c p}=\frac{N_{c p}}{\left(M+N_{P}\right)} E_{b}$ is the energy allocated to transmit the cyclic prefix and $N_{c p}$ is the number of subcarriers allocated to transmit the cyclic prefix.

Therefore, the total OFDM-DCSK energy to transmit one bit may be expressed as

$$
E_{\mathrm{tot}}=\left(\frac{N_{c p}+M+N_{P}}{M+N_{P}}\right) E_{b} .
$$

To study the energy efficiency, we compute the transmitted Data-energy-to-Bit-energy Ratio (DBR) which is defined as

$$
\mathrm{DBR} \equiv \frac{\mathrm{E}_{\text {data }}}{\mathrm{E}_{\text {tot }}} .
$$

Equation (18) gives the energy efficiency of the system by computing the ratio of the used energy to transmit the data signal to the total energy. Hence, a good energy efficiency for a system tends to one, i.e. $D B R \rightarrow 1$, which means that the total energy $E_{\text {tot }}$ is used to transmit the data, $E_{\text {data }}=E_{\text {tot }}$. Hence, in a conventional DCSK system, half of the energy is dissipated into the reference for each bit and the DBR is

$$
\mathrm{DBR}=\frac{1}{2}
$$

Therefore, by replacing equation (15) into equation (17), the DBR of the OFDM-DCSK system given in equation (18) becomes

$$
\mathrm{DBR}=\frac{\mathrm{M}}{\left(\mathrm{N}_{\mathrm{cp}}+\mathrm{M}+\mathrm{N}_{\mathrm{P}}\right)} .
$$

The DBR performance given in equation (20) is evaluated with and without the effect of cyclic prefix energy, for $N_{P}=3$ private subcarriers. The number of cyclic prefix subcarriers is set to $N_{c p}=4$ according to the IEEE 801.11a standard [35]. As shown in Fig. 4, the cyclic prefix energy reduces the energy efficiency of the system. Therefore, this $\mathrm{CP}$ is required in order to enhance the robustness of the system in multipath propagation environments. Moreover, Fig. 4 shows that for $M \leq 8$, the OFDM-DCSK yields lower or similar DBR than the DCSK system. Hence, using $M<8$ is not common in OFDM systems. For $M=8$, the OFDM-DCSK system is equivalent to DCSK system. In this case, $50 \%$ of the total bit energy $E_{t o t}$ is used to transmit the reference and cyclic prefix 


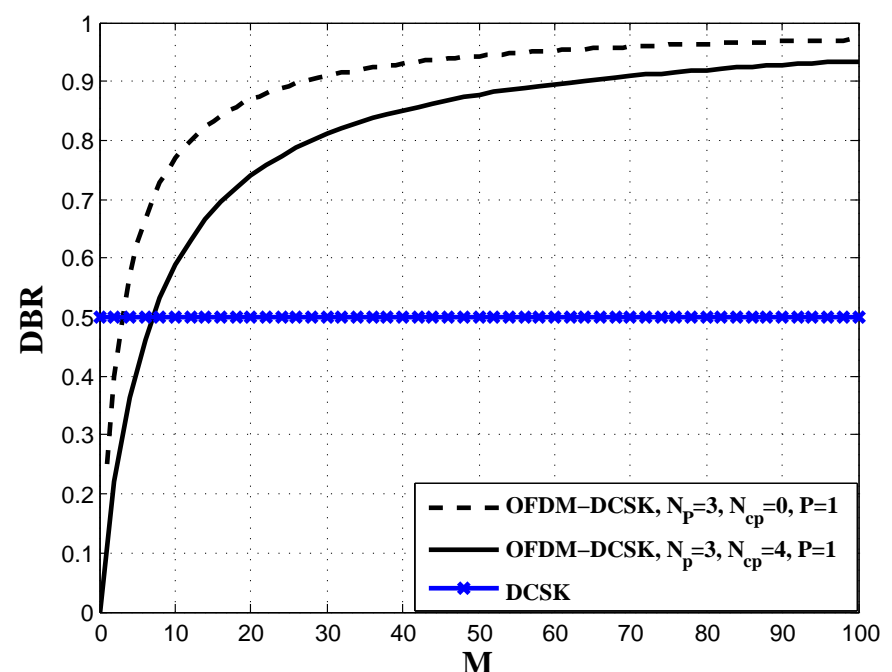

Fig. 4: $D B R$ versus number of data subcarriers $M$.

signals. The DBR of coherent systems is close to 1 because all the bit energy is used for bit transmission. Therefore, we can see that for $M>50$, reference and cyclic prefix energies account for less than $10 \%$ of the total energy $E_{t o t}$ for each bit in the $M$ data stream, a case in which the energy efficiency approaches the energy efficiency of coherent systems.

\section{E. The non-coherent receiver}

The block diagram of the MU OFDM-DCSK receiver is illustrated in Fig. 2. One of the objectives of this design is to reduce the implementation complexity by replacing the parallel matched filters used in [29] by simple FFT operations.

As shown in Fig. 2, the cyclic prefix is removed first, then an FTT operation is performed over every $N$ different samples which are then stored in two matrix memories $\mathbf{R}$ and $\mathbf{Y}$ dedicated for the reference and data signals respectively. Finally, after $\beta$ successive FFT operations, a DCSK demodulation is performed to recover the transmitted bits. Hence, the multi-user spread data $Y\left(k, i, f_{S_{p_{i}}}\right)$ and the reference signal of the $p^{\text {th }}$ user $R\left(k, f_{P_{p_{\nu}}}\right)$ after the $k^{\text {th }}$ FTT operation can be represented as

$$
\begin{gathered}
Y\left(k, i, f_{S_{p_{i}}}\right)=\sum_{p=1}^{P} s_{i, p} x_{k, u} H_{k, p, l, f_{S_{p_{i}}}}+N(s), \\
R\left(k, f_{P_{p_{\nu}}}\right)=x_{k, p} H_{k, p, l, f_{P_{p_{\nu}}}}+N(p),
\end{gathered}
$$

where $N(s), N(p), H_{p, f_{S_{p_{i}}}}$ and $H_{p, f_{P_{p_{\nu}}}}$ are the additive white Gaussian noises and the frequency channel responses of the $f_{S_{p_{i}}}^{\text {th }}$ public shared subcarrier and the $f_{P_{p_{\nu}}}^{\text {th }}$ private subcarrier, respectively. $R\left(k, f_{P_{p_{\nu}}}\right)$ contains the $k^{\text {th }}$ reference sample of user $p$ recovered from the private frequency $f_{P_{p_{\nu}}}$ and $Y\left(k, i, f_{S_{p_{i}}}\right)$ contains the multi-user $k^{\text {th }}$ sample of the $i^{\text {th }}$ bit transmitted over the shared $f_{S_{p_{i}}}$ frequency. Hence, the channel response at the subcarrier $f_{P_{p_{\nu}}}$ is given by

$$
H_{k, p, l, f_{P_{p_{\nu}}}}=\sum_{l=1}^{L_{p}} \alpha_{p, l, f_{P_{p_{\nu}}},\left\lceil\frac{k N T_{c}}{T_{h, p}}\right\rceil} e^{\left(-2 \pi j f_{P_{p_{\nu}}} \tau_{p}\right)}
$$

where $L_{p}$ is the number of channel paths of user $p$. In our system, the maximum delay spread for a given user $p$ is lower than the OFDM-DCSK symbol and its guard interval $\Delta$, i.e $\tau_{p, \max }<<\beta T_{c}$ and $\tau_{p, \max }<<<\Delta$. In this case, the channel considered in (7) can be seen by each set of OFDM subcarriers as flat and quasi static fading channel. In addition, the use of spreading spectrum technique along with interval guards makes the multipath interference neglected at the receiver side [36], [16]. Therefore, under the assumption of low maximum delay spread value, the term $e^{\left(-2 \pi j f_{P_{p_{\nu}}} \tau_{p}\right)} \approx 1$. Hence, the channel response simplifies to

$$
H_{k, p, f_{P_{p_{\nu}}}}=\sum_{l=1}^{L_{p}} \alpha_{p, l, f_{P_{p_{\nu}}},\left\lceil\frac{k N T_{c}}{T_{h, p}}\right\rceil} .
$$

Similarly, the channel response of the public frequency $f_{S_{p_{i}}}$ becomes

$$
H_{k, p, l, f_{S_{p_{i}}}}=\sum_{l=1}^{L_{p}} \alpha_{p, l, f_{S_{p_{i}}},\left\lceil\frac{k N T_{c}}{T_{h, p}}\right\rceil} .
$$

For lower values of the maximum delay spread, the channel is assumed to be flat in frequency over a couple of subcarriers. In the proposed comb-type design, $N_{P}$ replicas of reference signal are distributed in the OFDM spectrum to make the demodulation of the bits that lie between the private subcarriers and the edges of the spectrum possible. Therefore, the number $N_{P}$ can be increased or decreased depending on the coherence bandwidth of the channel. Thus, the following condition may be maintained for the $p^{\text {th }}$ user

$$
\alpha_{p, l, f_{S_{p_{i}}},\left\lceil\frac{k N T_{c}}{T_{h, p}}\right\rceil} \approx \alpha_{p, l, f_{P_{p_{\nu}}},\left\lceil\frac{k N T_{c}}{T_{h, p}}\right\rceil}
$$

Finally, the data of user $p$ are despread and decoded by computing the sign of the decision variable as follows

$$
D_{i, p}=\mathbb{R}\left\{\sum_{k=1}^{\beta} Y\left(k, i, f_{S_{p_{i}}}\right) \cdot R\left(k, f_{P_{p_{\nu}}}\right)^{*}\right\},
$$

where $\mathbb{R}$ designates the real part of the signal, $R\left(k, f_{P_{p_{\nu}}}\right)^{*}$ is the complex conjugate of the reference signal $R\left(k, f_{P_{p_{\nu}}}\right)$.

\section{Performance Analysis of MU OFDM-DCSK SYSTEM}

In this section, the performance of the MU OFDM-DCSK system is evaluated and the analytical BER expression is derived under multiple access interference in multipath Rayleigh fading channels.

To derive the analytical BER expression for a given user $p$, the mean and the variance for a given bit $i$ of the observation signal $D_{i, p}$ must be evaluated. With this aim, we start by mentioning some properties of chaotic signals which will be used later to analyse the statistical properties of the observation signal. As a matter of fact, a chaotic generator is very 
sensitive to initial conditions and we can deduce that different chaotic sequences generated from different initial conditions are independent from each other. In addition, the independence between the chaotic sequence and the Gaussian noise is also true [5]. For mathematical simplification throughout this work, we omit the use of the chip duration $T_{c}$ in this section.

The decision variable of equation (27) may be developed as

$$
\begin{aligned}
& D_{i, p}=\mathbb{R}\left\{s_{i, p} \sum_{l=1}^{L_{p}} \sum_{k=1}^{\beta}\left|\alpha_{p, l,\left\lceil\frac{k N}{T_{h, p}}\right\rceil}\right|^{2} x_{k, p}^{2}+\right. \\
& \underbrace{\sum_{l=1}^{L_{p}} \sum_{k=1}^{\beta} x_{p, k}\left(\alpha_{p, l,\left[\frac{k N}{T_{h, p}}\right\rceil}^{*} N_{S, k}+\alpha_{p, l,\left[\frac{k N}{T_{h, p}}\right\rceil} s_{p, i} N_{P, k}^{*}\right)}_{A}+ \\
& \underbrace{\sum_{k=1}^{\beta} N_{P, k}^{*} N_{S, k}}_{B}+ \\
& \underbrace{\sum_{l=1}^{L_{p}} \sum_{\substack{u=1 \\
u \neq p}}^{P} \sum_{l^{\prime}=1}^{L_{u}} \sum_{k=1}^{\beta} \alpha_{p, l,\left\lceil\frac{k N}{T_{h, p}}\right\rceil^{*}{ }_{u, l^{\prime},\left\lceil\frac{k N}{T_{h, u}}\right\rceil} s_{i, u} x_{k, p} x_{k, u}}+}_{C} \\
& \underbrace{\sum_{\substack{u=1 \\
u \neq p}}^{P} \sum_{l^{\prime}=1}^{L_{u}} \sum_{k=1}^{\beta} \alpha_{u, l^{\prime},\left\lceil\frac{k N}{T_{h, u}}\right\rceil} s_{i, u} x_{k, u} N_{P, k}^{*}}_{D}\} .
\end{aligned}
$$

The first term is the useful signal component while the terms $A$ and $B$ represent the additive noise interference and the terms $C$ and $D$ are MAI signals present in the decision variable. In addition, $\alpha_{p, l,\left[\frac{k N}{T_{h, p}}\right]}^{*}$ and $L_{p}$ are the complex conjugate of the channel coefficient and the number of paths for user $p$, respectively. Likewise, $L_{u}$ and $\alpha_{u, l^{\prime},\left[\frac{k N}{T_{h, u}}\right\rceil}$ are the number of paths and the channel coefficient for user $u$, respectively. In this paper, the Gaussian approximation is used to derive the performance of the MU OFDM-DCSK system.

Thus, the decision variable of MU OFDM-DCSK may be expressed as

$$
D_{i, p}=\frac{s_{i, p} M}{M+N_{P}} \sum_{l=1}^{L_{p}} \sum_{v=1}^{\chi_{p}}\left|\alpha_{p, l, v}\right|^{2} E_{b}+A+B+C+D
$$

where $E_{b}$ is the transmitted bit energy given in equation (14), $\chi_{p}$ represents the number of different channel coefficients during the MU OFDM-DCSK symbol of user $p$ explained in (7).

For an arbitrary $i^{\text {th }}$ bit and conditioned on the channel coefficients of the $p^{\text {th }}$ user useful signal $\sum_{l=1}^{L_{p}} \sum_{v=1}^{\chi_{p}} \alpha_{p, l, v}$, the instantaneous mean and variance of the decision variable are derived as follows

$$
E\left(D_{u, i, z}\right)=s_{i, p} \frac{M}{M+N_{P}} \sum_{l=1}^{L_{p}} \sum_{v=1}^{\chi_{p}}\left|\alpha_{p, l, v}\right|^{2} E_{b} .
$$

Since all the terms of equation (29) are uncorrelated and independent with zero mean, the variance of each term is equal to the expectation of its squared value. Hence, the conditional variance of the decision variable for an arbitrary $i^{\text {th }}$ bit is given by

$$
V\left(D_{i}\right)=E\left(A^{2}\right)+E\left(B^{2}\right)+E\left(C^{2}\right)+E\left(D^{2}\right),
$$

where $V(\cdot)$ indicates variance.

Provided that the channel coefficients are independent with zero mean and the terms $N_{P, k}^{*} N_{S, k}$ and $x_{p, k}$ are also independent and uncorrelated, the conditional variance of the term $A$ will be

$$
V(A)=\frac{M E_{b}}{M+N_{P}} N_{0} \sum_{l=1}^{L_{p}} \sum_{v=1}^{\chi_{p}}\left|\alpha_{p, l, v}\right|^{2} .
$$

The variance of $B$ will be

$$
V(B)=\frac{\beta N_{0}^{2}}{4} .
$$

The term $C$ is the sum of multiple access interference signals. Based on SGA which invokes the central limit theorem, this MAI signal can be approximated as an additive white Gaussian noise additional to the background noise [32], [33]. Based on the SGA assumption and the above uncorrelation and independence conditions, the general expression of the conditional variance of $C$ will be

$$
\begin{aligned}
& V(C)= \\
& \sum_{l=1}^{L_{p}} \sum_{v=1}^{\chi_{p}}\left|\alpha_{p, l, v}\right|^{2} \sum_{\substack{u=1 \\
u \neq p}}^{P} \sum_{l^{\prime}=1}^{L_{u}} \sum_{v^{\prime}=1}^{\chi_{u}} E\left(\left|\alpha_{u, l^{\prime}, v^{\prime}}\right|^{2}\right) \sum_{k=1}^{\beta} E\left(x_{k, p}^{2} x_{k, u}^{2}\right) .
\end{aligned}
$$

In our paper we assume that chaotic sequences have a unity variance $E\left(x^{2}=1\right)$. Since chaotic sequences are independent, the expectation of the product $E\left(x_{k, p}^{2} x_{k, u}^{2}\right)$ is equal to $E\left(x_{k, p}^{2}\right)$. Substituting equation (14) into equation (34) will result in the variance of $C$

$$
\begin{aligned}
& V(C)= \\
& \frac{M E_{b}}{M+N_{P}} \sum_{l=1}^{L_{p}} \sum_{v=1}^{\chi_{p}}\left|\alpha_{p, l, v}\right|^{2} \sum_{\substack{u=1 \\
u \neq p}}^{P} \sum_{l^{\prime}=1}^{L_{u}} \sum_{v^{\prime}=1}^{\chi_{u}} E\left(\left|\alpha_{u, l^{\prime}, v^{\prime}}\right|^{2}\right) .
\end{aligned}
$$
The term $\sum_{\substack{u=1 \\ u \neq p}}^{P} \sum_{l^{\prime}=1}^{L_{u}} \sum_{v^{\prime}=1}^{\chi_{u}} E\left(\left|\alpha_{u, l^{\prime}, v^{\prime}}\right|^{2}\right)$ represents the general form of the MAI signal generated from the different
channel path gains of different users. This term may be simplified in some special cases, for example if the channel gains of all paths for all users are equal then this term reduces to $(P-1) \chi_{u} L_{u} E\left(\left|\alpha_{u, l^{\prime}, v}\right|^{2}\right)$. Nonetheless, we prefer to keep it in its general form paraded by (35) in this paper.

Finally, the variance of $D$ could be obtained as

$$
V(D)=\frac{M E_{b} N_{0}}{2\left(M+N_{p}\right)} \sum_{\substack{u=1 \\ u \neq p}}^{P} \sum_{l^{\prime}=1}^{L_{u}} \sum_{v^{\prime}=1}^{\chi_{u}} E\left(\left|\alpha_{u, l^{\prime}, v^{\prime}}\right|^{2}\right) .
$$


In order to compute the BER with our approach, the error probability must be first evaluated for a given received energy $E_{b}^{(u)}$ and channel coefficients of the useful signal $\sum_{l=1}^{L_{p}} \sum_{v=1}^{\chi_{p}} \alpha_{p, l, v}$. Considering the bit energy as a deterministic variable, the decision variable at the output of the correlator is necessarily a Gaussian random variable. Using equations (30) and (31), the bit error probability of the $p^{\text {th }}$ user would be expressed in the form

$$
\begin{aligned}
& \mathrm{BER}= \\
& \frac{1}{2} \operatorname{Pr}\left(D_{i, p}<0 \mid s_{i, p}=+1\right)+\frac{1}{2} \operatorname{Pr}\left(D_{i, p}>0 \mid s_{i, p}=-1\right) \\
& \quad=\frac{1}{2} \operatorname{erfc}\left(\frac{\mathrm{E}\left(\mathrm{D}_{\mathrm{i}, \mathrm{p}} \mid \mathrm{s}_{\mathrm{i}, \mathrm{p}}=+1\right)}{\sqrt{2 \mathrm{~V}\left(\mathrm{D}_{\mathrm{i}, \mathrm{p}} \mid \mathrm{s}_{\mathrm{i}, \mathrm{p}}=+1\right)}}\right)
\end{aligned}
$$

where $\operatorname{erfc}(\cdot)$ is the complementary error function defined by

$$
\operatorname{erfc}(\mathrm{x}) \equiv \frac{2}{\sqrt{\pi}} \int_{\mathrm{x}}^{\infty} \mathrm{e}^{-\mu^{2}} \mathrm{~d} \mu .
$$

Rearranging the terms above results in the following BER expression for the MU OFDM-DCSK system

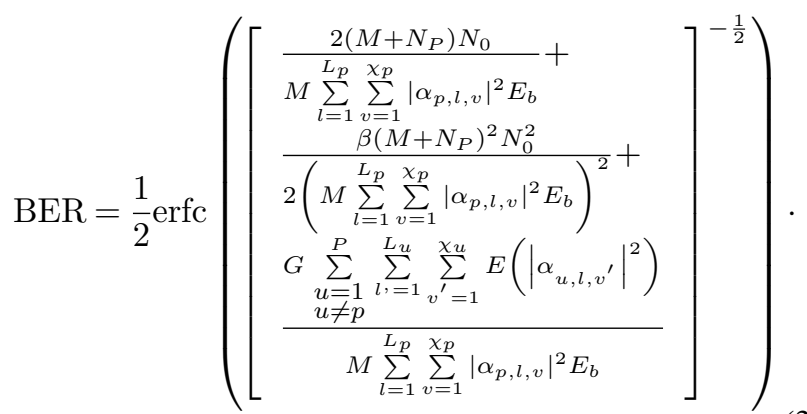

where $G=\left(M+N_{P}\right)\left(2+N_{0}\right)$. This overall BER expression may be simplified as

$$
\mathrm{BER}=\frac{1}{2} \operatorname{erfc}\left(\left[\frac{2}{\gamma}+\frac{\beta}{2 \gamma^{2}}+\frac{1}{\rho}\right]^{-\frac{1}{2}}\right),
$$

where $\gamma$ and $\rho$ represent the the instantaneous signal-tonoise ratios generated from the AWGN and MAI components respectively. These two components may be explicitly shown to be

$$
\begin{gathered}
\gamma=\frac{M \sum_{l=1}^{L_{p}} \sum_{v=1}^{\chi_{p}}\left|\alpha_{p, l, v}\right|^{2} E_{b}}{\left(M+N_{P}\right) N_{0}} . \\
\rho=\frac{M \sum_{l=1}^{L_{p}} \sum_{v=1}^{\chi_{p}}\left|\alpha_{p, l, v}\right|^{2} E_{b}}{G \sum_{\substack{u=1 \\
u \neq p}}^{P} \sum_{l^{\prime}=1}^{L_{u}} \sum_{v^{\prime}=1}^{\chi_{u}} E\left(\left|\alpha_{u, l, v^{\prime}}\right|^{2}\right)} .
\end{gathered}
$$

Equations (40) and (41) are both functions of the bit energy $E_{b}$. As shown in Fig. 5, the bit energy $E_{b}$ cannot be assumed constant once the bit is spread by the chaotic sequence. In fact, because of the non-periodic nature of chaotic signals, the emitted bit energy after spreading will definitely vary from one bit to another for low spreading factors [9]. In addition, the histogram of Fig. 5 has been obtained using one million samples. From these samples, energies of successive bits are calculated for the given spreading factor. The bit energy is assumed to be the output of a stationary random process [37]. The histogram obtained in Fig. 5 can be considered as a good estimation of the probability density function (pdf) of $E_{b}$. Based on this property and specifically for low spreading factors, the resultant pdf of $\gamma$ and $\rho$ becomes equivalent to the pdf of the sum of channel gains multiplied by the pdf of energy distribution.

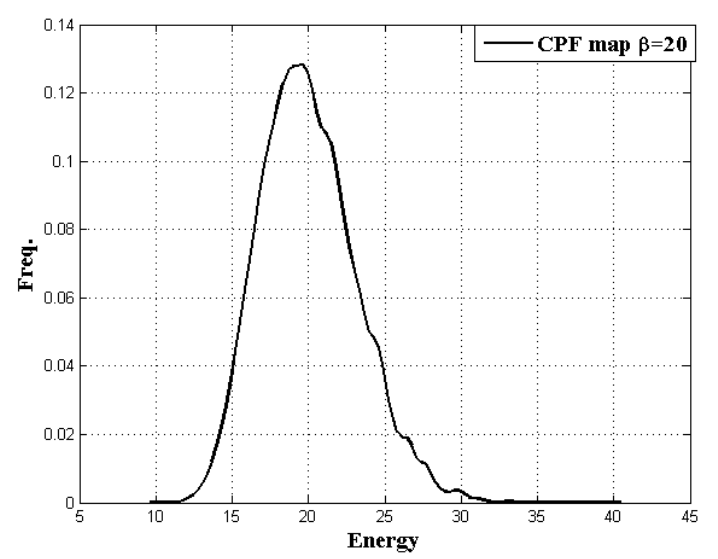

Fig. 5: Histogram of the distribution of $E_{b}$ for $\beta=20$.

Finally, the average BER expression for MU OFDM-DCSK under multipath fading channels would be expressed as

$$
\overline{\mathrm{BER}}=\frac{1}{2} \int_{0}^{\infty} \int_{0}^{\infty} \operatorname{erfc}\left(\left[\frac{2}{\gamma}+\frac{\beta}{2 \gamma^{2}}+\frac{1}{\rho}\right]^{-\frac{1}{2}}\right) f(\gamma) f(\rho) d \gamma d \rho .
$$

It is to be noted that the closed form expression of the PDF of $E_{b}$ is difficult to obtain [9]. The two random variables in $\gamma$ (see equations (40) and (41)) are the multiplication of the channel gains and the bit energy $E_{b}$ which make the analytical derivation of $\gamma$ and $\rho$ intractable. To overcome this issue, the resultant pdf may be obtained by plotting the histogram of these random variables which can be considered as a good estimation of the probability density function of $\gamma$ and $\rho$. Moreover, since it is hard to obtain a closed-form solution to the double integral given in equation (42), we have used numerical integration to compute the average BER stated here.

\section{A. Special case: BER computation methodology under AWGN channel}

In this section, the performance of the OFDM-DCSK under an AWGN channel will be evaluated for low and high spreading factors. The aim of this analysis is to highlight the non-constant bit energy problem when the spreading factor is very low. For AWGN scenario, $\gamma$ and $\rho$ given in equations (40) and (41) simplify to 


$$
\begin{gathered}
\gamma=\frac{M E_{b}}{\left(M+N_{P}\right) N_{0}} . \\
\rho=\frac{M(P-1) E_{b}}{G} .
\end{gathered}
$$

Hence, for high spreading factors, $E_{b}$ can be considered constant [9] and the BER may be approximated as

$$
\mathrm{BER}=\frac{1}{2} \operatorname{erfc}\left(\left[\begin{array}{l}
\frac{2\left(M+N_{P}\right) N_{0}}{M E_{b}}+ \\
\frac{\beta\left(M+N_{P}\right)^{2} N_{0}^{2}}{2 M^{2} E_{b}^{2}}+ \\
\frac{G(P-1)}{M E_{b}}
\end{array}\right]^{-\frac{1}{2}}\right) .
$$

For low spreading factors, $E_{b}$ cannot be constant and the average BER of MU OFDM-DCSK becomes

$$
\overline{\mathrm{BER}}=\frac{1}{2} \int_{0}^{\infty} \operatorname{erfc}\left(\left[\begin{array}{l}
\frac{2\left(M+N_{P}\right) N_{0}}{M E_{b}}+ \\
\frac{\beta\left(M+N_{P}\right)^{2} N_{0}^{2}}{2 M^{2} E_{b}^{2}}+ \\
\frac{G(P-1)}{M E_{b}}
\end{array}\right]^{-\frac{1}{2}}\right) p\left(E_{b}\right) d E_{b} .
$$

Given the shape of the bit energy distribution, the analytical expression appears difficult to compute, leaving numerical integration as a solution for performing the BER computation of equation (46).

\section{Simulation Results}

In this section the bit error rate performance of MU OFDMDCSK is evaluated over AWGN and Rayleigh fading channels for different number of transmitted bits $M$, spreading factor lengths $\beta$, number of channel coefficients $\chi$ per OFDM-DCSK symbol and number of users $P$.

Simulation parameters for the system under consideration are set according to the IEEE 801.11 $a$ standard [35] where 25 $\%$ of the total subcarriers $N_{t}$ are unused, (i.e $N_{u}=25 \% N_{t}$ ), $N_{c p}$ is equal to 4 and the size of FFT is chosen to accommodate the number of users and keep the system flexible to change the number of data $M$, where $M \leq N_{S}$ given in equation (6). It is important to note that among $N_{S}$ subcarriers, the leftover subcarriers, i.e. the ones not used in transmission are nullified in order not to degrade the system performance. Finally, the parameters of the proposed MU OFDM-DCSK system are chosen as tabulated next.

\begin{tabular}{|l|l|}
\hline Parameters & \\
\hline FFT size & Variable \\
\hline Subcarrier spacing & $312.5 \mathrm{KHz}$ \\
\hline FFT Sampling frequency & $\begin{array}{l}(312.5 \mathrm{KHz}) \\
\times \text { FFT size }\end{array}$ \\
\hline Cylcic prefix duration & $T_{c p}=0.8 \mu s$ \\
\hline Number of private frequencies $N_{P}$ & 3 \\
\hline Total OFDM symbol duration & $T_{\text {OFDM } \mu s}$ \\
\hline Spreading factor $\beta$ & Variable \\
\hline Total DCSK-OFDM symbol duration & $T_{\text {OFDM }} \times \beta$ \\
\hline Number of users & Variable \\
\hline
\end{tabular}

As shall be clearly observed throughout this section, full accordance and conformity between simulation results and computed BER expressions with respect to the bit energy $E_{b}$ is achieved for all studied scenarios, which indicates the veracity or our analytical work. On the other hand, the use of CP elongates the time symbol and increases the total transmitted energy. This causes the causes the decrement of data rate and the degradation of system performance. Therefore, the reduction in the data rate and the signal-to-noise ratio is equal to $N /\left(N+N_{c p}\right)$ and $S N R_{\text {lost }}=10 \log _{10}\left(1-\mathrm{N} / \mathrm{N}_{\mathrm{cp}}\right)$ respectively. In other terms, the energy $E_{c p}$ used to transmit the $\mathrm{CP}$ introduces a constant degradation in performance, i.e. a gap in the communication system equal to $10 \log _{10}\left(\mathrm{E}_{\mathrm{cp}}\right) \mathrm{dB}$.

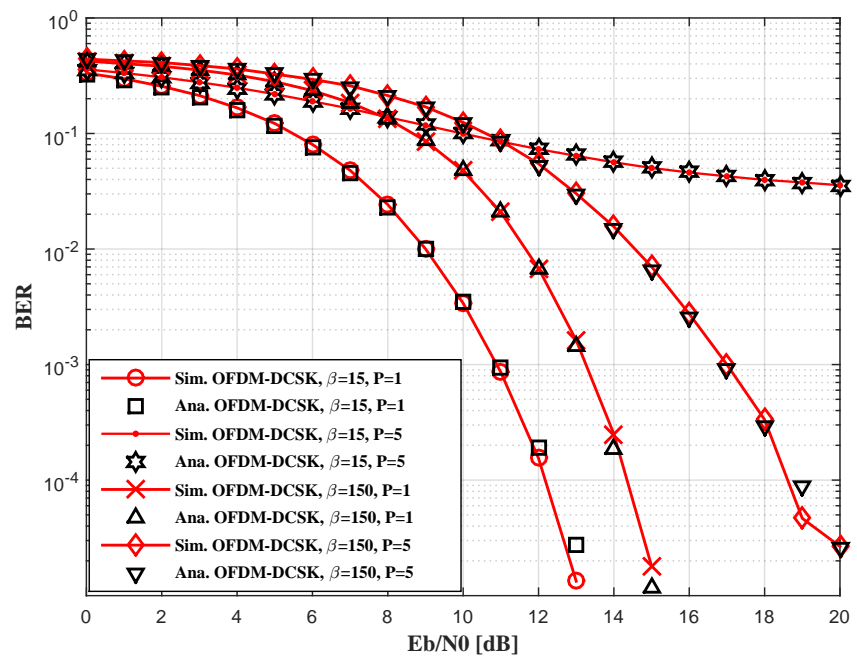

Fig. 6: Simulation and analytical BER performance over AWGN channel of MU OFDM-DCSK system for different spreading factor values $(\beta=15,150), M=49$ and $N_{t}=128$ with $P=1$ and $P=5$ users.

Simulation and analytical BER performances based on (45) and (46) for an FFT size equal to $N_{t}=128, M=49$ bits per OFDM-DCSK symbol, $\beta=15,150$ for the cases of one and five users in AWGN channels are shown in Fig 6. We observe in the same figure that for the single-user scenario, the performance of OFDM-DCSK system at a spreading factor $\beta=15$ is superior to that at $\beta=150$. This is because in this class of non-coherent modulation over AWGN channels, the reference and information bearing signals are both corrupted by the channel noise and at the receiver, a noisy reference signal is correlated with a noisy information bearing signal, consequently, this deteriorates the performance of the DCSK system. As a matter of fact, longer sequence lengths are expected to deteriorate the performance in general under such circumstances. The problem of optimal code length is widely studied in [5], [38].

Hence, the reverse of the spreading factor length is observed in multi-user case, as observed in Fig. 6, MAI caused by the additional users in the same bandwidth for the case of $\beta=$ 15 introduces a huge performance degradation. Reduction of MAI can be achieved by increasing the spreading factor at the expense of increased MU OFDM-DCSK symbol time. The degradation observed in Fig. 6 is somehow compensated by using a higher spreading value of $\beta=150$. To answer the 
question of optimal spreading factor length in presence of MAI signal, Fig. 7 plots the BER over AWGN channel against the spreading factor values for different number of users and for $M=49, N_{t}=128$ and $E_{b} / N_{0}=12 \mathrm{~dB}$. We can clearly see that the optimal spreading factor $\beta$ in the single-user case is shifted to higher values when the number of users increases. In particular, the minimum values of BER are obtained with the approximate values of $\beta$ equal to $5,25,40$ and 50 for $P=1,2,5,8$ users respectively. These results mean that good performances are obtained at moderate values of spreading factor, in other words, the proposed system can perform well even with a short OFDM-DCSK time symbol.

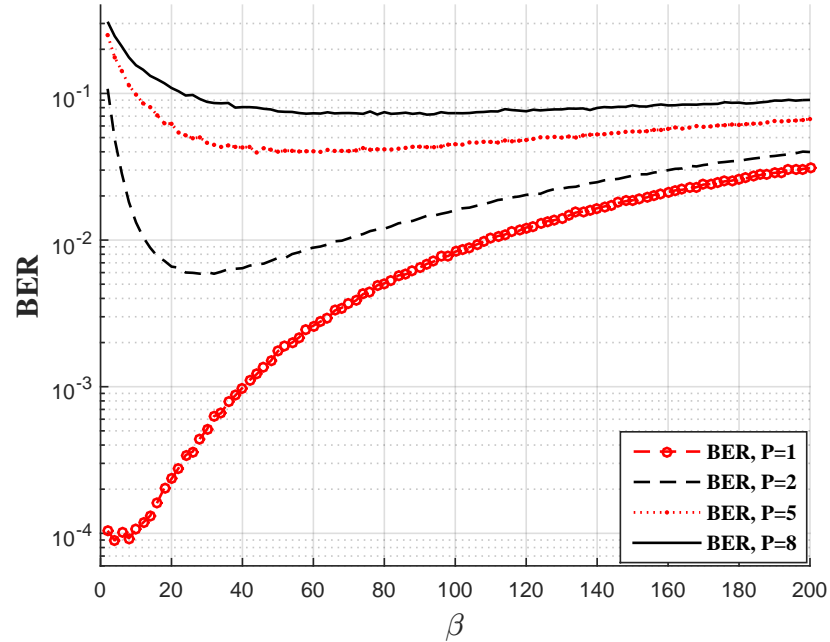

Fig. 7: BER values against the spreading factor $\beta$ for $E_{b} / N_{0}=12$ $\mathrm{dB}, N_{t}=128$ and $M=49$.

Fig. 8 shows the effect of number of subcarriers on the BER performance in AWGN channels. To this end, a single-user system, $P=1$, with the following parameters is simulated: $M=3$ and 17 for an FFT size equal to $N_{t}=32$ and $M=49$ bits for an FFT size equal $N_{t}=128$ and spreading factor $\beta=15$. As seen in Fig. 8, the increment of $N_{t}$ has enhanced the performance, this is because such increment causes the DBR ratio to approach unity which means that for a higher number of subcarriers $M$, less energy is needed to reach a given BER. Note that in the case of $M=3$, the OFDMDCSK system is equivalent to the conventional DCSK system. Hence, the results obtained at $M=3$ could form a sort of BER comparison between the proposed system and the DCSK. In addition, we observe a small performance enhancement between $M=17$ and $M=49$. This small increment in performance beyond certain value of $M$ is due to the marginal difference of the two DBR values corresponding to the given values of $M=17$ and $M=49$. Fig. 4 shows that DBR starts to saturate beyond certain values of $M$. The performance of MU OFDM-DCSK in multipath Rayleigh fading channels is evaluated and presented in Fig. 9 for $M=49$ bits per user an FFT size equal $N_{t}=128$ and various values of the spreading factor, i.e. $\beta=12$ and $\beta=120$. The maximum delay spread of this channel is $\tau_{L_{p}}=0.1 \mu \mathrm{s}$ which makes this latter flat in

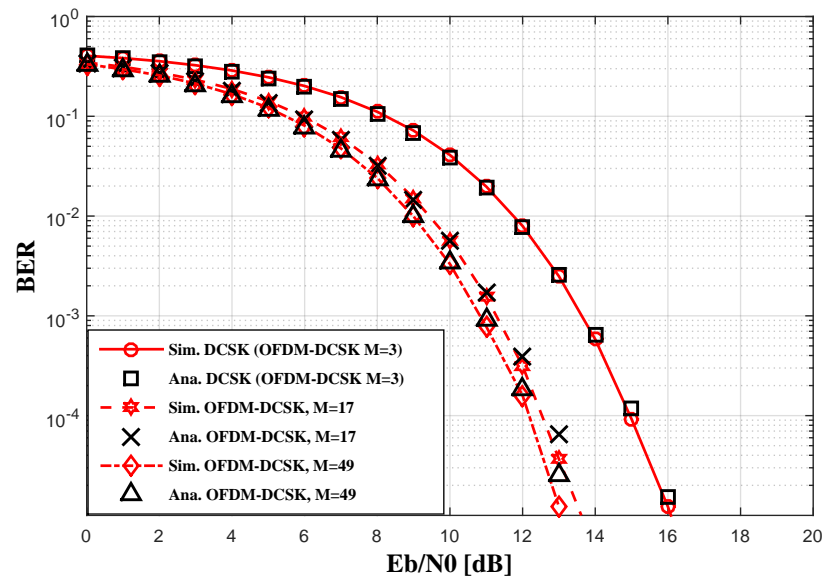

Fig. 8: BER comparison of the single-user (i.e $P=1$ ) OFDM-DCSK for $M=17,49$ and DCSK (i.e $M=3$ ) for $N_{t}=32,128$ and a spreading factor $\beta=15$ under AWGN.

frequency over a bandwidth of $10 \mathrm{Mhz}$. With this configuration, 3 reference signals can properly cover the entire OFDM spectrum and make the demodulation possible. The analytical results shown in these plots are based on equation (42). Every user channel has 3 independent paths and a unity average gain. In addition, the channel coefficients are maintained constant during 3 OFDM symbol times, $\chi=3$. Besides confirming our arguments related to multi-user OFDM-DCSK scenarios, the results obtained in Fig. 9 confirm once again the fact that MAI can be mitigated using higher spreading factor values.

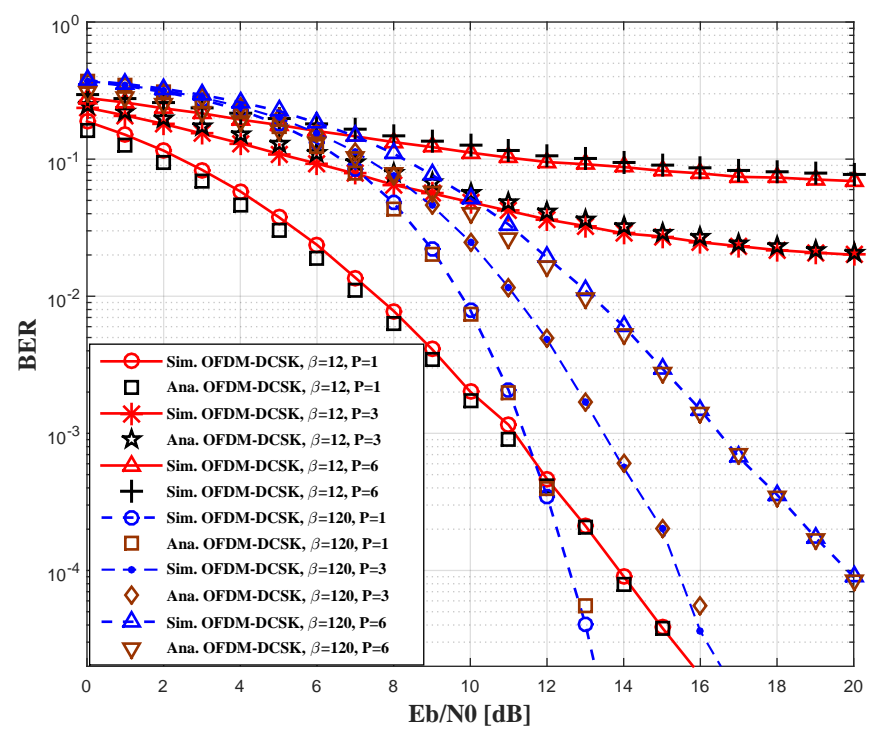

Fig. 9: Simulation and analytical BER performance of MU OFDMDCSK for $\beta=12,120, M=49, N_{t}=128$ in multipath Rayleigh fading channels with $L_{p}=3, \chi=3$, and equal average power gain $E\left(\alpha_{p}^{2}\right)=1$ for $P=1,3$ and 6 users.

In order to evaluate the extent to which the proposed design with comb-type arrangement of the reference signal exploits the time diversity of the wireless channel, we simulate 


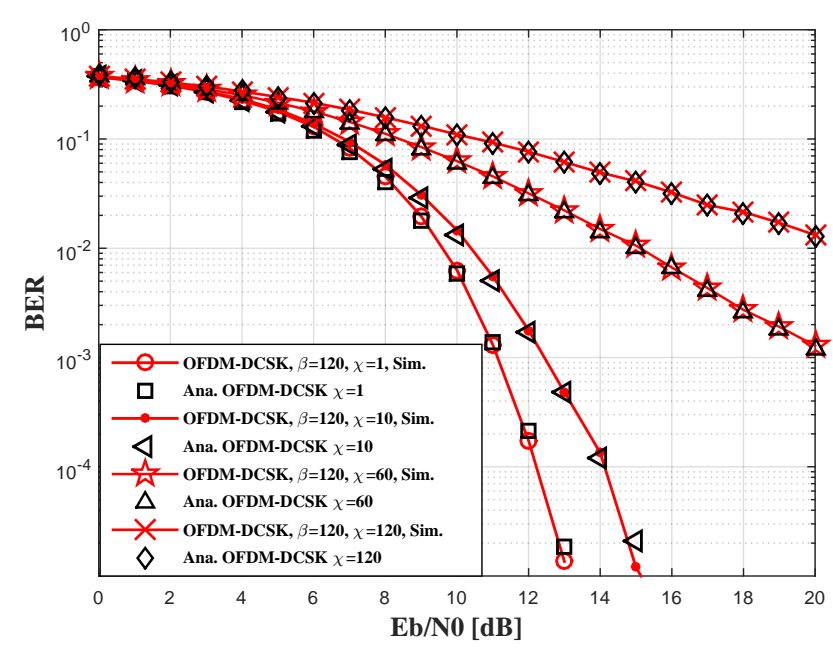

Fig. 10: Simulation and analytical BER performance of single-user OFDM-DCSK exploiting the time diversity for $\beta=120, M=49$ and $N_{t}=128$ in multipath Rayleigh fading channels with $L_{p}=3$, average power gain $E\left(\alpha_{p}^{2}\right)=1$ and $\chi=1,10,60,120$.

a single-user OFDM-DCSK system with a spreading factor $\beta=120$ and different values of $\chi$. Fig. 10 shows the BER performance for the four different values of $\chi=1,10,60$ and 120. As observed in Fig. 10, the system shows the best performance at $\chi=1$ because channel coefficients change $\beta$ times during the OFDM-DCSK symbol in this case. In fact, every transmitted OFDM-DCSK bit will have $\beta$ different channel coefficients and the time diversity order of this system reaches its maximum and equals the spreading factor $\beta$. The worst BER performance is obtained when no time diversity is observed, a situation which occurs when channel coefficients are constant during a single OFDM-DCSK bit transmission period which is expressed by $\chi=120$. In Figs. 6-10 the excellent conformity between simulation results and analytical BER expressions confirms the accuracy of our multi-user scenario derivations.

The BER performance comparison between the proposed MU OFDM-DCSK, OFDM-DCSK [30] and MC-DCSK [19] systems is given in Fig. 11. With similar bandwidth consumption for the three systems, $M=49$ and an FFT size equal $N_{t}=128$, the transmission is done over multipath Rayleigh channel with 3 paths, $\chi=\beta$, and the maximum delay spread is equal to $\tau_{L p}=0.1 \mu \mathrm{s}$. Hence, with this delay spread, the coherence bandwidth of the channel is roughly equal to 10 Mhz. Since the coherence bandwidth is higher than the used spectrum, using only one reference signal will be insufficient to demodulate all the bits. In addition, the simulation is carried out for the single-user scenario, $P=1$, because the OFDMDCSK presented in [30] and the MC-DCSK do not support the multi-user scenario. Simulation results show that our proposed OFDM-DCSK system outperforms the OFDM-DCSK [30] and MC-DCSK by 3 and $7 \mathrm{~dB}$ respectively at the value of $\mathrm{BER}=4 \cdot 10^{-2}$. This is due to the fact that reference signals distributed on the two edges and at the center of the spectrum allow better recovering of the $M$ bits at different frequencies

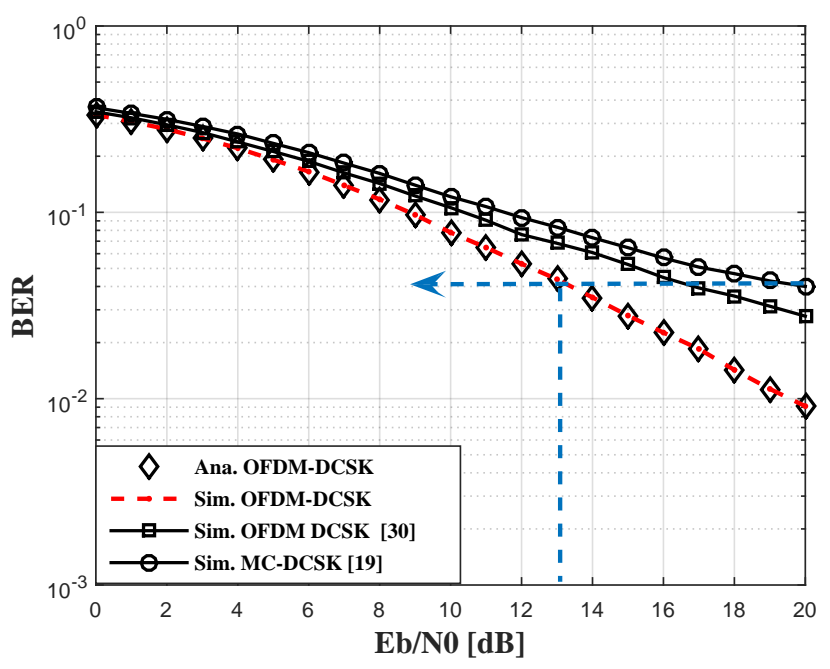

Fig. 11: Comparison between the proposed, MC-DCSK [19] and OFDM-DCSK [30] systems for $M=49$ and $N_{t}=128$.

and channel gains. In OFDM-DCSK systems, however, a single reference signal is implemented at the center of the spectrum. This disables the correct recovery of the bits at the edges since the channel gains at the edges are different from those at the center. The same argument is valid in evaluating the performance of MC-DCSK where central bits are not well recovered because the reference signal is located at the edge of the spectrum.

\section{Conclusions}

A multi-user OFDM-DCSK has been proposed in this paper. This new system aims at increasing the spectral and energy efficiencies, allowing multiple access transmission, reducing complexity by using IFFT/FFT operations instead of parallel matched filters as in MC-DCSK and solving the RF delay line problem faced in conventional DCSK schemes. The key element of this design is to assign $N_{P}$ private subcarriers to each user and leave the remaining $N_{S}=N-P N_{P}$ subcarriers as shared public subcarriers. The private subcarriers are used to transmit the reference signals of the users, while the public subcarriers are shared with other users to carry data. For any individual user, only $N_{P}$ replicas of the chaotic reference signal are used to transmit $M$ bits, instead of using $M$ reference signals as done in DCSK system $\left(N_{p}<<M\right)$. The energy efficiency of the proposed system is analysed and a DBR is derived. Our results indicate that for $M>50$ subcarriers, the energy loss in transmitting the reference signal is less than $10 \%$ of the total bit energy. The performance of the proposed system is studied and bit error rate expressions for AWGN and multipath Rayleigh fading channels are derived. Simulation results being matched to theoretical BER expressions affirms our derivation approach. In addition, the obtained results highlight the importance of the comb-type design to exploit the time diversity of wireless channels. To compare the performance of the proposed system to that of DCSK, MC-DCSK and OFDM-DCSK, the simulated BERs 
are plotted where results show a performance enhancement in the proposed system compared to rival systems. Considering the need and demand of future wireless communications to multiuser communications at minimized bandwidth and energy costs, the proposed OFDM-DCSK system is promising.

\section{REFERENCES}

[1] T. S. Rappaport, Wireless Communications: Principles and Practice. Englewook Cliffs, NJ: Prentice-Hall, 1996.

[2] L. Hanzo, T. Keller, M. Muenster, and B.-J. Choi, OFDM and MCCDMA for Broadband Multi-User Communications, WLANs and Broadcasting. New York, NY, USA: John Wiley \& Sons, Inc., 2003.

[3] R. V. Nee and R. Prasad, OFDM for Wireless Multimedia Communications, 1st ed. Norwood, MA, USA: Artech House, Inc., 2000.

[4] S. Kondo and B. Milstein, "Performance of multicarrier DS-CDMA systems," IEEE Trans. Commun., vol. 44, no. 2, pp. 238 -246, Feb 1996.

[5] F. C. M. Lau and C. K. Tse, Chaos-Based Digital communication systems. Springer-Verlag, 2003.

[6] A. P. Kurian, S. Puthusserypady, and S. M. Htut, "Performance enhacment of DS-CDMA system using chaotic complex spreading sequence," IEEE Trans. Wireless Commun., vol. 4, no. 3, pp. 984-989, May 2005.

[7] R. Vali, S. Berber, and S. K. Nguang, "Accurate derivation of chaosbased acquisition performance in a fading channel," IEEE Trans. Wireless Commun., vol. 11, no. 2, pp. 722 -731, february 2012.

[8] S. Vitali, R. Rovatti, and G. Setti, "Improving PA efficiency by chaosbased spreading in multicarrier DS-CDMA systems," in Proc. 2006 IEEE International Symposium on Circuits and Systems, (ISCAS), May 2006, pp. 1194-1198.

[9] G. Kaddoum, P. Chargé, and D. Roviras, "A generalized methodology for bit-error-rate prediction in correlation-based communication schemes using chaos," IEEE Commun. Letters., vol. 13, no. 8, pp. 567-569, 2009.

[10] G. Kaddoum, P. Chargé, D. Roviras, and D. Fournier-Prunaret, "A methodology for bit error rate prediction in chaos-based communication systems," Birkhäuser, Circuits, Systems and Signal Processing, vol. 28, pp. 925-944, 2009.

[11] Y. Fang, L. W. J. Xu, and G. Chen, "Performance of MIMO relay DCSKCD systems over nakagami fading channels," IEEE Trans. on Circuits and Syst. I: Reg. Papers, vol. 60, pp. 757-767, March 2013.

[12] Y. Fang, L. Wang, and G. Chen, "Performance of a multiple-access DCSK-CC system over nakagami-m fading channels," in Proc. 2013 IEEE International Symposium on Circuits and Systems (ISCAS), May 2013, pp. 277-280.

[13] W. Xu, L. Wang, and G. Chen, "Performance of DCSK cooperative communication systems over multipath fading channels," IEEE Trans. on Circuits and Syst. I: Reg. Papers, vol. 58, no. 1, pp. 196 -204, Jan. 2011.

[14] J. Xu, W. Xu, L. Wang, and G. Chen, "Design and simulation of a cooperative communication system based on DCSK/FM-DCSK," in Proc. 2010 IEEE International Symposium on Circuits and Systems (ISCAS), June 2010, pp. $2454-2457$.

[15] L. M. Pecora, T. L. Carroll, and G. A. Johson, "Fundamentals of synchronization in chaotic systems, concepts, and applications," International journal of Bifrurcation and Chaos, vol. 74, pp. 520-543, 1997.

[16] P. Chen, L. Wang, and F. Lau, "One analog STBC-DCSK transmission scheme not requiring channel state information," IEEE Trans. Circuits and Syst. I: Reg. Papers, vol. 60, no. 4, pp. 1027-1037, 2013.

[17] G. Kaddoum, J. Olivain, G. Beaufort Samson, P. Giard, and F. Gagnon, "Implementation of a differential chaos shift keying communication system in gnu radio," in International Symposium on Wireless Communication Systems (ISWCS), 2012, pp. 934 - 938.

[18] Y. Xia, C. K. Tse, and F. C. M. Lau, "Performance of differential chaosshift-keying digital communication systems over a multipath fading channel with delay spread," IEEE Trans. Circuits and Syst. II: Exp. Briefs, vol. 51, pp. 680-684, 2004.

[19] G. Kaddoum, F. Richardson, and F. Gagnon, "Design and analysis of a multi-carrier differential chaos shift keying communication system," IEEE Trans. on Commun.,, vol. 61, no. 8, pp. 3281-3291, 2013.

[20] C.-C. Chong and S. K. Yong, "UWB direct chaotic communication technology for low-rate WPAN applications," IEEE Trans. Veh. Technol., vol. 57, no. 3, pp. 1527-1536, May 2008.

[21] S. Wang and X. Wang, "M-DCSK-Based chaotic communications in MIMO multipath channels with no channel state information," IEEE Trans. on Circuits and Syst. II: Exp. Briefs, vol. 57, no. 12, pp. 10011005, Dec. 2010.
[22] G. Kaddoum, F. Gagnon, P. Charge, and D. Roviras, "A generalized ber prediction method for differential chaos shift keying system through different communication channels," Wireless Personal Communications, vol. 64, pp. 425-437, 2012.

[23] W. K. Xu, L. Wang, and K. G., "A novel differential chaos shift keying modulation scheme," International Journal of Bifurcation and Chaos, vol. 21, no. 03, pp. 799-814, 2011.

[24] G. Kaddoum and F. Gagnon, "Design of a high data rate differential chaos-shift keying system," IEEE Trans. on Circuits and Syst. II: Exp. Briefs, vol. 59, no. 99, pp. 1-5, Jul. 2012.

[25] H. Yang and G. ping Jiang, "High-efficiency differential-chaos-shiftkeying scheme for chaos-based non coherent communication," IEEE Trans. on Circuits and Syst. II: Exp. Briefs, vol. 59, no. 5, pp. 312316, 2012.

[26] H. Yang and G.-P. Jiang, "High-efficiency differential-chaos-shift-keying scheme for chaos-based noncoherent communication," IEEE Trans. Circuits and Syst. II: Exp. Briefs, vol. 59, no. 5, pp. 312 -316, May 2012.

[27] S. Wang and X. Wang, "M-DCSK based chaotic communications in MIMO multipath channels with no channel state information," IEEE Trans. on Circuits and Syst. II: Exp. Briefs, vol. 57, no. 12, pp. 10011005, Dec 2010.

[28] P. Chen, L. Wang, and G. Chen, "DDCSK-Walsh coding: A reliable chaotic modulation-based transmission technique," IEEE Trans. on Circuits and Syst. II: Exp. Briefs, vol. 59, no. 2, pp. 128-132, Feb. 2012.

[29] G. Kaddoum, F. Gagnon, and F.-D. Richardson, "Design of a secure multi-carrier DCSK system," in Proc. 2012 The ninth international symposium on wireless communication systems (ISWCS), June 2012, pp. 964-968.

[30] S. Li, Y. Zhao, and Z. Wu, "Design and analysis of an OFDMbased differential chaos shift keying communication system," Journal of Communications, vol. 3, pp. 199-205, 2015.

[31] Y. Shen and E. Martinez, "Channel estimation in ofdm systems," Freescale Semiconductor, 2006.

[32] D. Borth and M. Pursley, "Analysis of direct-sequence spread-spectrum multiple-access communication over rician fading channels," IEEE Trans. on Commun., vol. 27, no. 10, pp. 1566-1577, Oct 1979.

[33] C. Gardner and J. Orr, "Fading effects on the performance of a spread spectrum multiple access communication system," IEEE Trans. on Commun., vol. 27, no. 1, pp. 143-149, Jan 1979.

[34] A. Nayak and I. Stojmenovic, Wireless Sensor and Actuator Networks:Algorithms and Protocols for Scalable Coordination and Data Communication, 1st ed. Wiley-IEEE Press, 2010.

[35] "IEEE standards association," http://standards.ieee.org/getieee802/ download/802.11a-1999.pdf.

[36] Y. Mostofi and D. Cox, "ICI mitigation for pilot-aided OFDM mobile systems," IEEE Trans. on Wireless Commun., vol. 4, no. 2, pp. 765-774, March 2005.

[37] S. H. Isabelle and G. W. Wornell, "Statistical analysis and spectral estimation techniques for one-dimensional chaotic signals," IEEE Trans. Sigal Proces., vol. 45, pp. 1495-1497, 1997.

[38] M. Sushchik, L. S. Tsimring, and A. R. Volkovski, "Performance analysis of correlation-based communication schemes utilizing chaos," IEEE Trans. Circuits and Syst. I: Reg. Papers, vol. 47, pp. 1684-1691, 2000 .

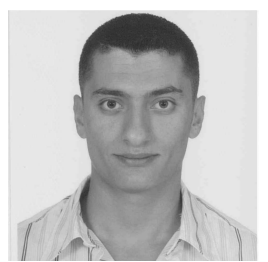

Georges Kaddoum (M'11) received the Ph.D. degree (with honors) in signal processing and telecommunications from the National Institute of Applied Sciences (INSA), University of Toulouse, Toulouse, France, in 2009. Since November 2013, he is an Assistant Professor of electrical engineering with the École de Technologie Supérieure (ETS), University of Quebec, Montréal, QC, Canada. His recent research activities cover wireless communication systems, chaotic modulations, secure transmissions, and space communications and navigation. He has published over 70 journal and conference papers and has two pending patents. Dr. Kaddoum received the Best Paper Award at the 2014 IEEE International Conference on Wireless and Mobile Computing, Networking, and Communications (WIMOB), with three coauthors, and the 2015 IEEE Transactions on Communications Top Reviewer Award. 